LA, ${ }^{\prime} 96-44,60$

TITLE: GLOBAL POSITIONING AUTOMATIC VEHICLE LOCATION SYSTEM

AUTHOR(S): BILL J. PAPATHEOFANIS, NIS-9

MARVIN L. HASENACK, NIS-9

ROBERT T. TELLER, FSS -20

GERALD F. RAMSEY, FSS-20

TO $\mathrm{BE}$

SUBMITTED TO DOE OFFICE OF SCIENTIFIC AND TECHNICAL INFORMATION (OSIT)

DISTRIITTON OF THIS DOCUENT IS UNLMITED
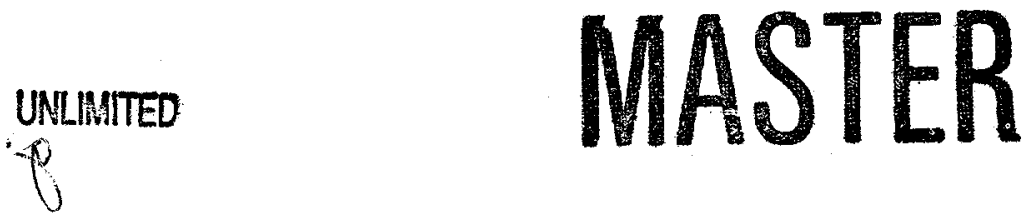

By acceptance of this article, the publisher recognizes that the U.S Government retains a nonexclusive, royalty-free license to publish or reproduce the published form of this coniribution. or 10 allow others to do so. for US Government purposes

The Los Alamos National Laboratory requests that the publisher identily this articie as work performed under the auspices ot the U S Department of Energy

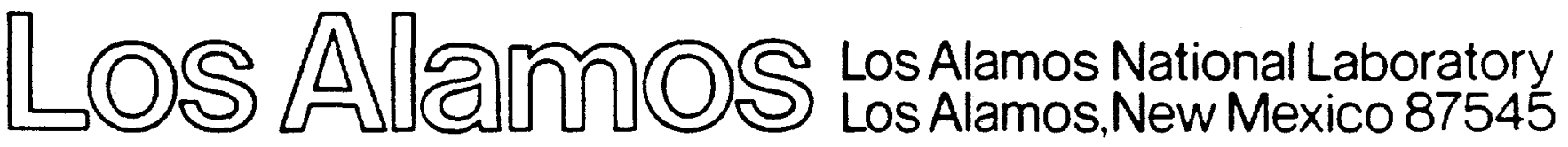


Title:

Global Positioning Automatic Vehicle Location System

\section{Authors:}

Bill J. Papatheofanis, NIS-9

Marvin L. Hasenack, NIS-9

Robert T. Teller, FSS-20

Gerald F. Ramsey, FSS-20

Submitted to:

DOE Office of Scientific and Technical Information (OST) 


\section{DISCLAMER}

Portions of this document may be illegible in electronic image products. Images are produced from the best available original document. 


\title{
Global Positioning System Automatic Vehicle Location System
}

\author{
Bill J. Papatheofanis, Marvin L. Hasenack, Robert T. Teller, \\ and Gerald F. Ramsey
}

\begin{abstract}
Los Alamos National Laboratory (LANL) is a unique facility covering over 43 square miles. The Emergency Management and Response Office (EM\&R) is required to respond, provide Incident Command (IC), and coordination for all Laboratory emergencies. This requires IC's and support staff to respond to the actual scene of the incident. Since the IC is under numerous constraints and stress, the office wanted the capability of locating the EM\&R vehicles on an electronic map. An automated vehicle location (AVL) system was required for the additional safety of the emergency response personal.

The requirements for the AVL system include total automatic tracking and low cost. After careful consideration, it was determined that the most efficient and cost effective system would be based on packet radio technology as the transmission media. The location is determined by the Department of Defense Global Positioning System (GPS).

The system that was designed and constructed required four components to be interfaced and communicate with each other. The first component was a GPS receiver which actually provides the location information, equipped with a digital interface to communicate location information remotely. The second component is a modem that interfaces the GPS digital interface information to a radio. The third component is the radio itself which allows for the actual information transfer from the remote GPS receiver and modem. The fourth component is the software package that provides moving maps and displays the vehicle location on that map.

The equipment was all commercial off-the-shelf that only required proper integration and packaging for the AVL application. This paper describes the steps taken in the integration of the equipment into the AVL package.
\end{abstract}




\section{Introduction}

Los Alamos National Laboratory (LANL) is a unique facility covering over 43 square miles. The Emergency Management and Response (EM\&R) office has the overall responsibility for coping with and mitigating the effects of any emergency at LANL. The EM\&R office supplies an Incident Command (IC) which responds to all laboratory emergencies. This requires the IC and support staff to respond to the actual scene of an incident. The EM\&R office wanted the capability of locating their vehicles on an electronic map because of the lack of markings on facilities, unmarked secondary roads, unmarked trails, and the lack of marked terrain features. This automated location system would provide the additional safety for the IC's in cases where something adverse happens to the responders. Many times the EM\&R IC is required to respond to a scene at night or during adverse weather conditions. With the automatic location system the IC can be directed to the scene even if land features are not available. The vehicle can also be located if the responder is incapacitated for any reason.

The automatic vehicle location (AVL) system is based on the Department of Defense Global Position System (GPS). The satellite based GPS system provides information to special receivers which collect data from several satelites and are then processed by a built in navigational computer. The GPS receiver then displays its location to within 100 feet in a selectable coordinate system. In addition to providing $x-y$ coordinates the receiver calculates altitude, speed, direction of travel, date and time.

The simplest method of vehicle tracking using GPS would be to install a receiver in a vehicle, and using a two-way radio, transmit the displayed coordinates to a dispatcher who can take the received coordinates and manually plot them on a paper map. Today's technology enables this process to be totally automated. This paper discusses how to put together a totally automated system using commercial off-theshelf equipment.

\section{EM\&R Vehicles}

EM\&R has two response vehicles which are equipped with AVL. Figure 1 is a photo of the 1994 Chevrolet Suburban which is the primary response vehicle. Figure 2 is a photo of the 1994 Jeep Cherokee

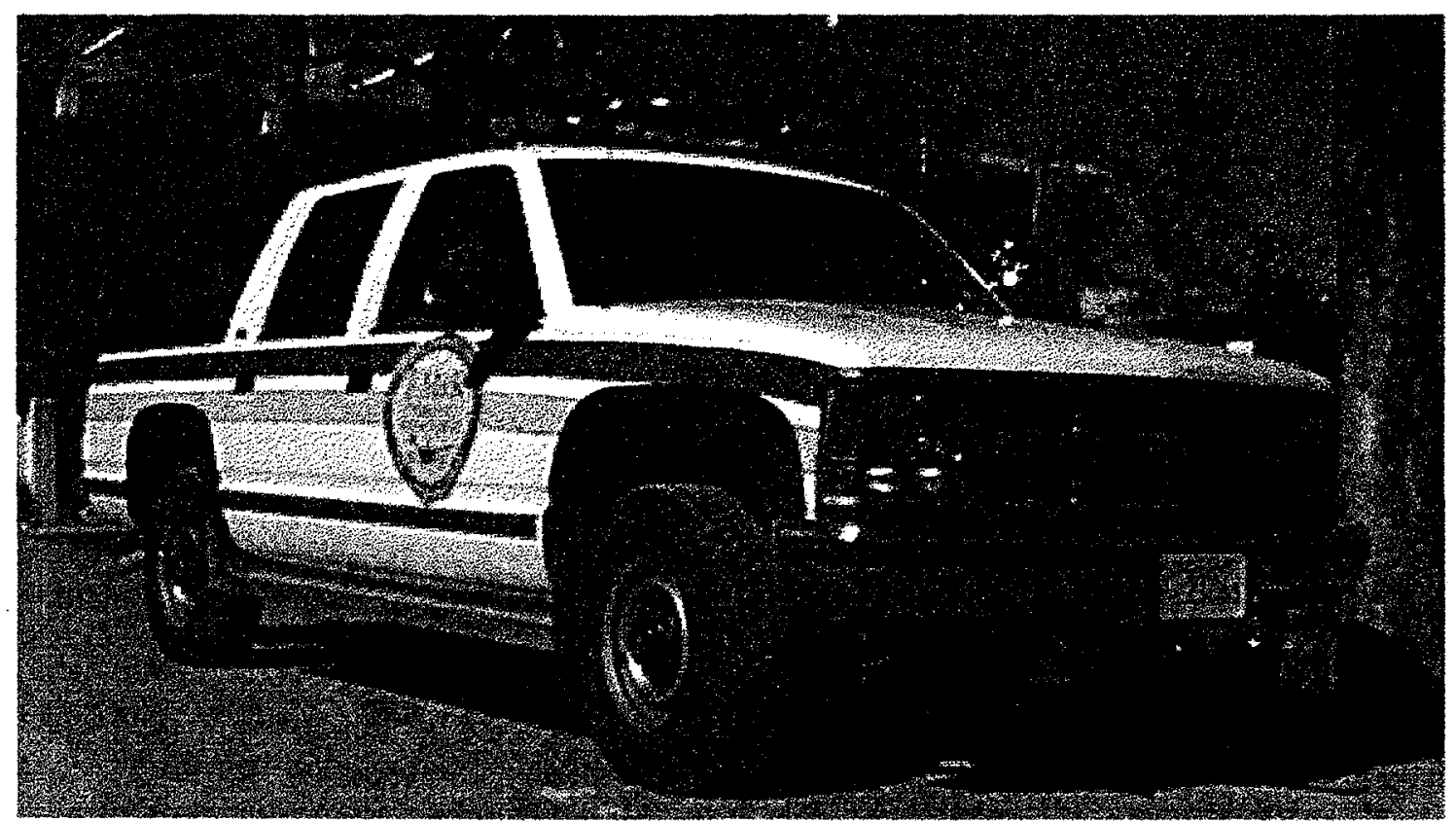

Figure 1 
which is the back-up vehicle that provides field support for the primary vehicle. Both vehicles carry numerous radios and cellular telephone for communications with the Emergency Operations Center (EOC) and multi agencies which respond to emergencies at LANL. The vehicles also carry documentation on Laboratory information required for the IC's to carry out their function.

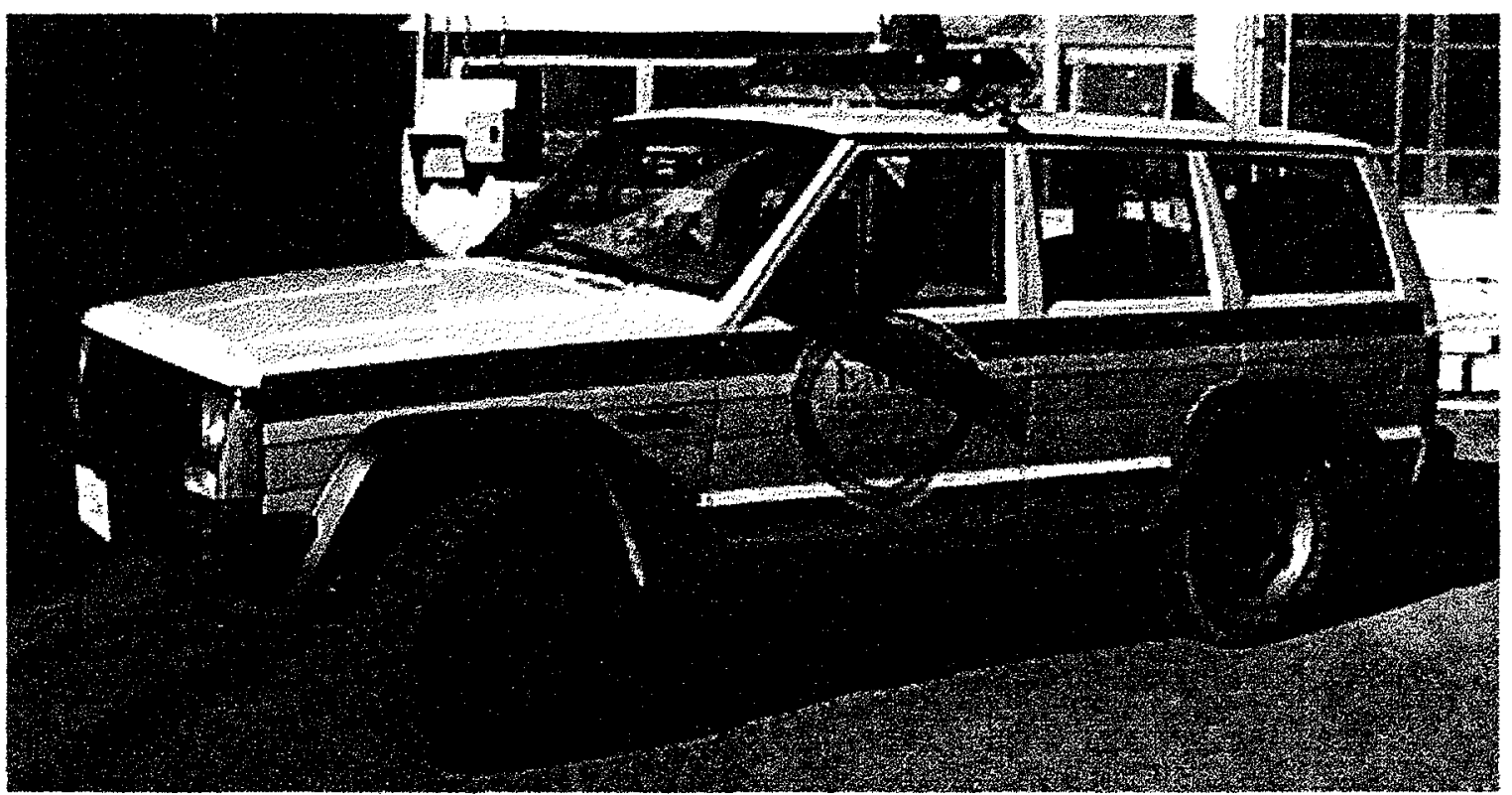

Figure 2

\section{Vehicle AVL Package}

The vehicle AVL package consists of three components. The first is the GPS unit, the second is the modem, and the third is the radio. In this section we will describe each component, the required specifications, and which manufacture's equipment was used (see Appendix 1 for addresses and phone numbers).

A. The GPS unit used is a Trimble Scout Master which met all the following requirements:

1. External Antenna - The GPS receiver requires an outside remote antenna to work properly when mounted inside a vehicle.

2. External Power - The GPS receiver should have external power capability. This allows the unit to get power directly from the vehicle and also provides a way of controlling when the receiver is powered on and off.

3. Serial Port - This allow the GPS receiver to communicate its position information with an external device via RS-232.

4. NEMA 183 Protocol - The output of the GPS receiver's serial port is programmable for various standard and proprietary protocols. NEMA 183 is the National Electrical Marine Associations 183 standard protocol which has become a standard in the GPS world. Most commercial mapping software recognizes the NEMA 183 protocol as well as do most radio modems. 
B. The radio modem used is a Kantronics WM1200 packet radio modem. This modem met all of the following requirements:

1. Packet Radio Standard AX.25 Protocol - This protocol provides a standard transmission method which is specifically written for radio networking and provides error correction.

2. Radio Interface - This modem is specifically manufactured to be connected to a radio.

3. GPS Ready - The WM1200 has built-in software specifically written to interpret NEMA 183 sentences from a GPS receiver and then broadcast them over the radio.

4. Unique Identification - Each modem has a unique programmable identification call sign. The call sign allows each vehicle to have a unique address.

5. Programmable Reporting - The modem allows the user to program vehicle reporting intervals allowing the system optimization.

C. The radio used was a Motorola Maxtrac 300. This radio was selected because of the following features:

1. External programmable interface - The Motorola Maxtrac 300 radio has a 16 pin external programmable interface. This interface is programmable through the radios programming software which allows the user to program the functionality of each pin on the 16 pin connector. This feature allows the modem to interface with the correct internal radio circuits to function most efficiently. This also allows for all the wiring to be external to the radio: no wires hang out from the inside of the radio that could provide interference paths to the radio and the modem.

2. Multiple encode/decode capability - The Motorola Maxtrac 300 is capable of generating and detecting all standard coded squelch signaling. This allows the radio to provide the necessary signaling to access the repeater. This also allows the same radio channel to be used for both data and voice without the two modes interfering with each other.

\section{Repeater}

The LANL system required a repeater for reliable coverage over the 43 square mile complex and additional areas outside the Laboratory. The repeater is required due to the propagation of VHF/UHF radio being limited to line of sight. As LANL terrain varies from mountain top to canyon bottom, the repeater was located on top of Pajarito Mountain which overlooks most of the Laboratory. The repeater is a Motorola Quantar Repeater which is a standard repeater station. The standard voice repeater was chosen over a digital repeater to avoid the hidden station problem. Digital repeaters rely on store forward technology which allows the repeater to transmit and receive on a single frequency. The digital repeater does not retransmit received signal simultaneously. It actually receives data, stores the data in a buffer, and then transmits the data from the buffer. The store forward repeater can hear all the stations on the network, but they cannot hear each other. The hidden station problem occurs when all the stations cannot hear each other, allowing multiple stations to try to transmit at the same time causing interference. 


\section{Base Station}

The base station is located at the EOC and consists of four components. The first two are the same as in the vehicle package: the radio and the modem. The third component is a computer which is capable of running the software to interpret the received data and display that data on a map. The computer for this installation is an IBM PC. The fourth component is the software itself. The software package that we have implemented on the system is from ERM. ERM software is specifically written to interpret NEMA 183 sentences and display this information an a moving map. The ERM software is very sophisticated and provides not only location information but is capable of displaying and recording multiple vehicle's positions at the same time.

\section{Vehicle Configuration}

The equipment was selected and initial tests were performed on the bench. When it was time to integrate the components into a vehicular package, the first attempt was under the premise that the vehicle users should have access to the GPS unit so that they know their position information as well as the same equipment performing the AVL function. This did work for a short period of time, however, problems with the AVL started to occur which were traced back to the GPS unit being set to erroneous parameters. Everyone that saw the GPS unit in the vehicle had to press all the buttons which placed the unit in an unusable operating state. At that point, we decided that the system should operate autonoumsly and the equipment should be packaged in a "Black Box" without any user selectable options. Figure 3 is an assemble diagram of the "Black Box". Figure 4 is a photo of the inside of one of the actual boxes.

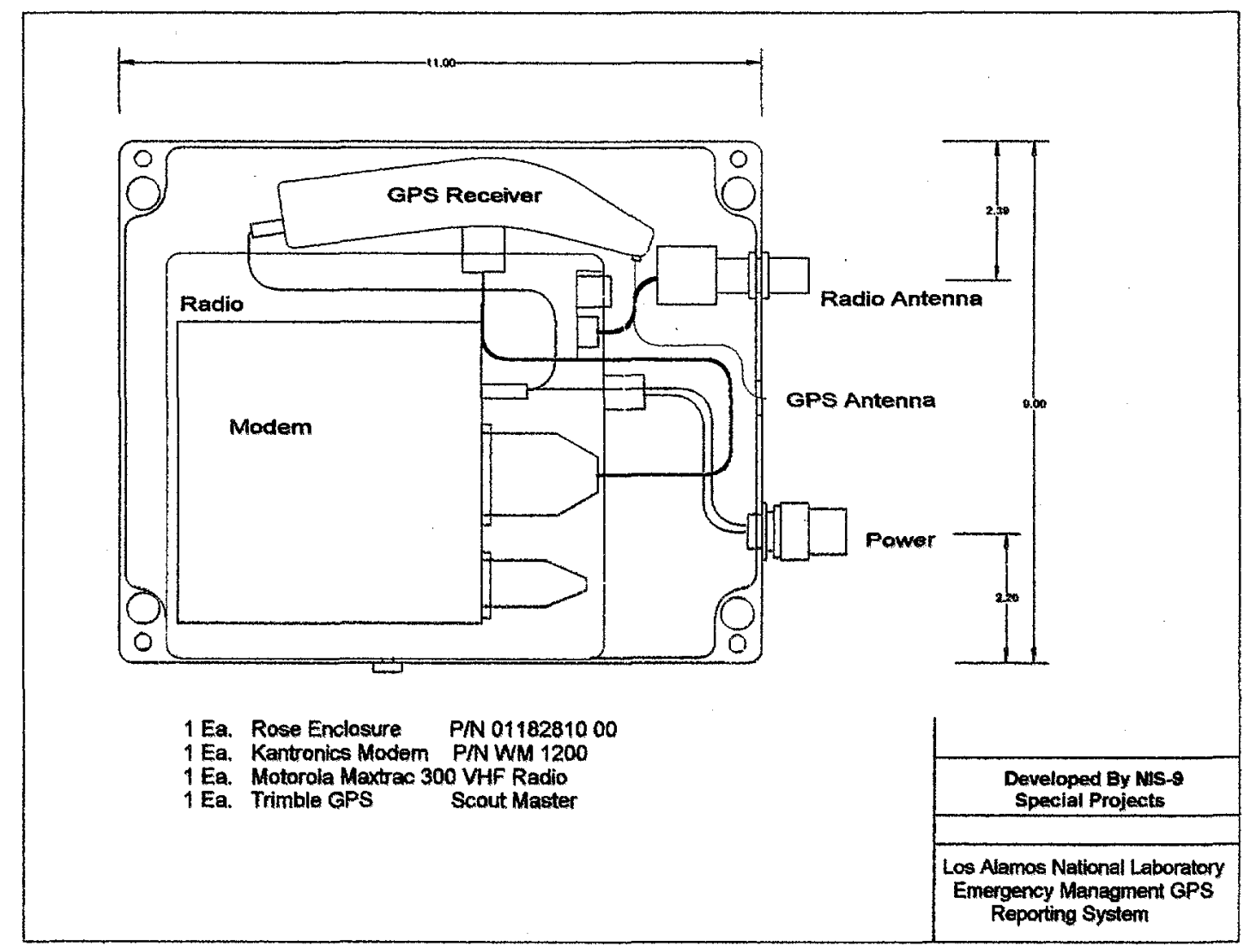

Figure 3 


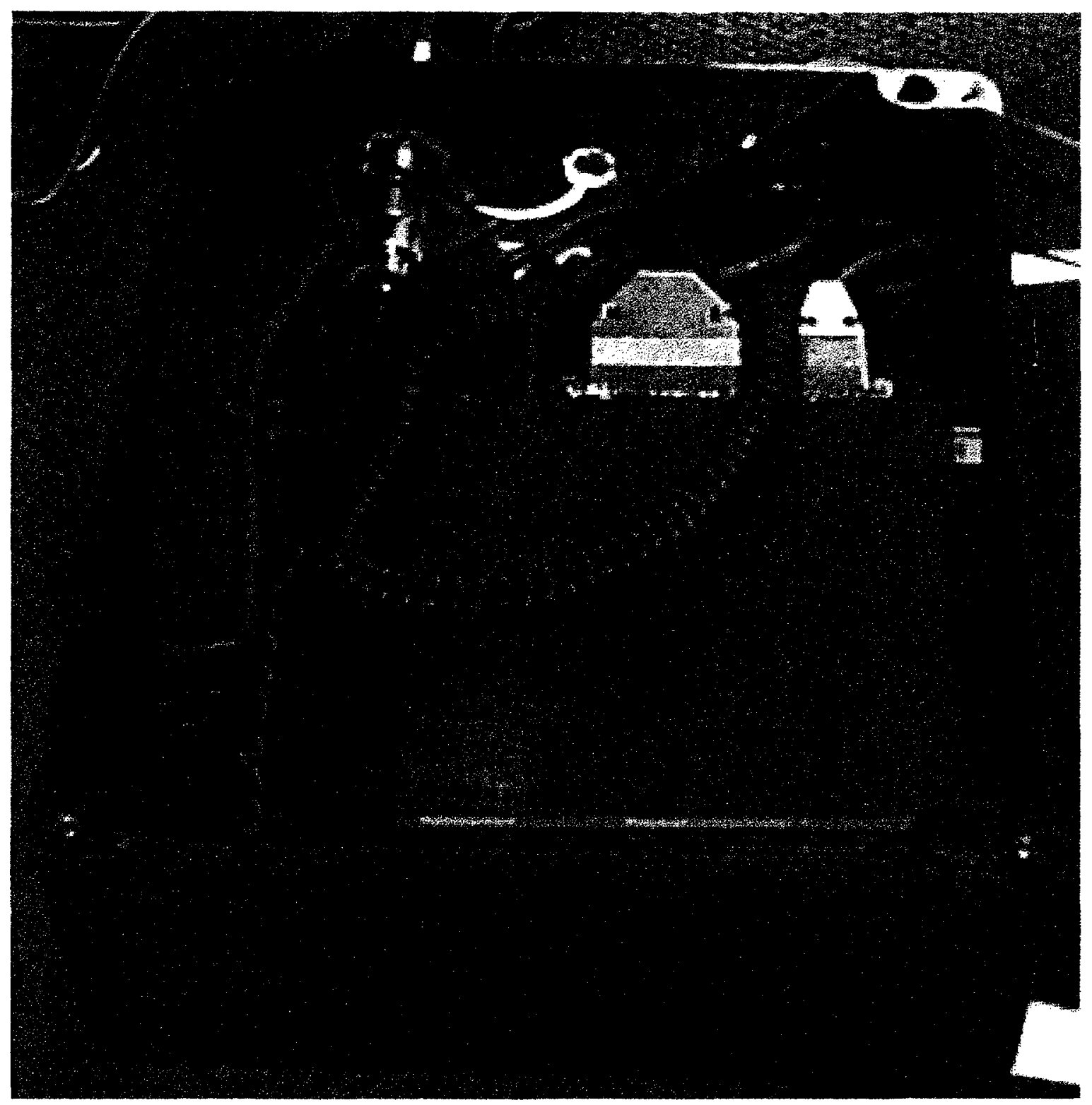

Figure 4

The next issue was where to put the boxes in both of the vehicles so that they were protected from daily vehicle use. In the Suburban, the "Black Box" was located underneath the pull out drawer that is in the cargo compartment of the vehicle (see Figure 5). In the Jeep, the "Black Box" was mounted in the rear cargo compartment next to one of the other radios (see Figure 6). 


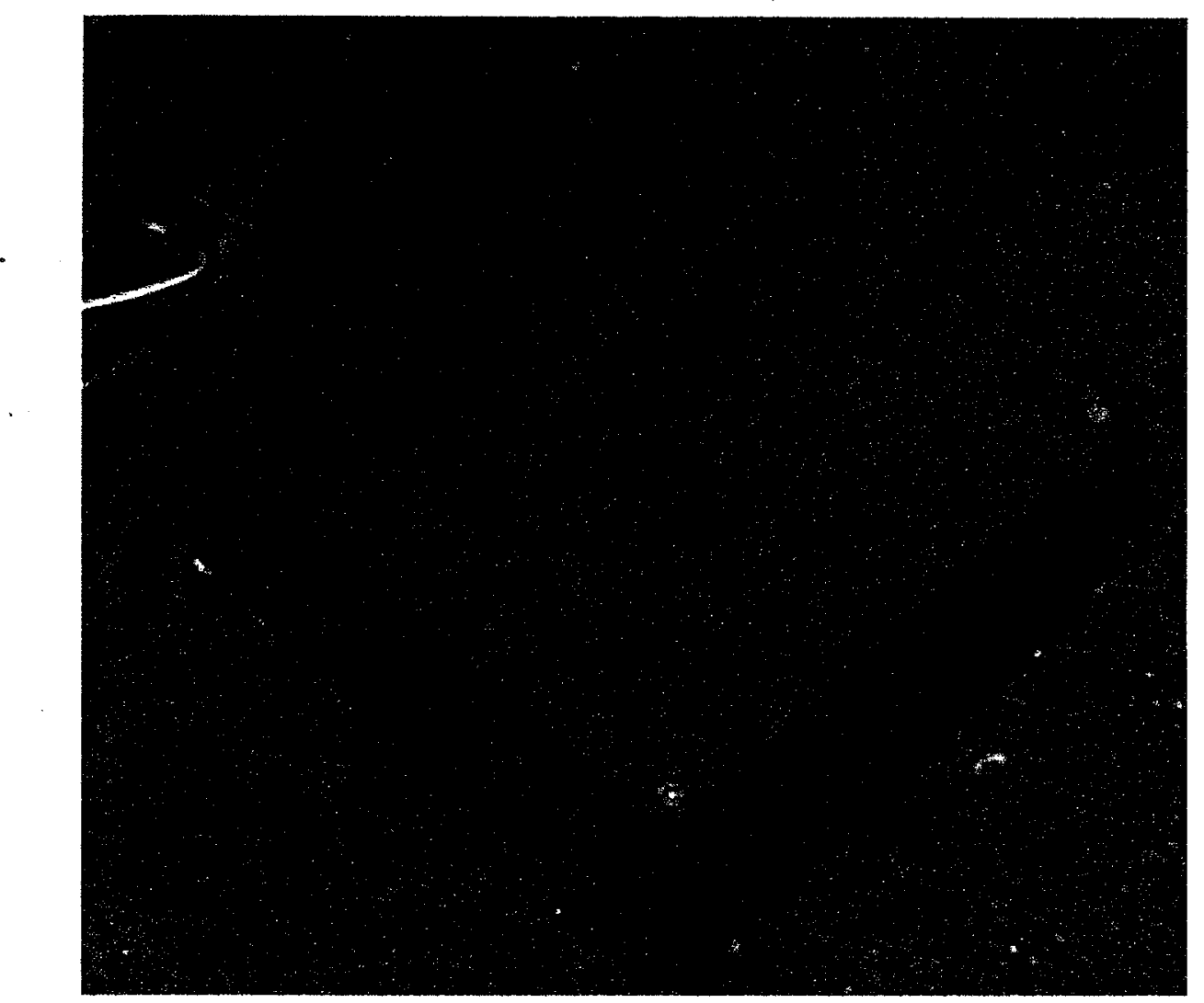

Figure 5

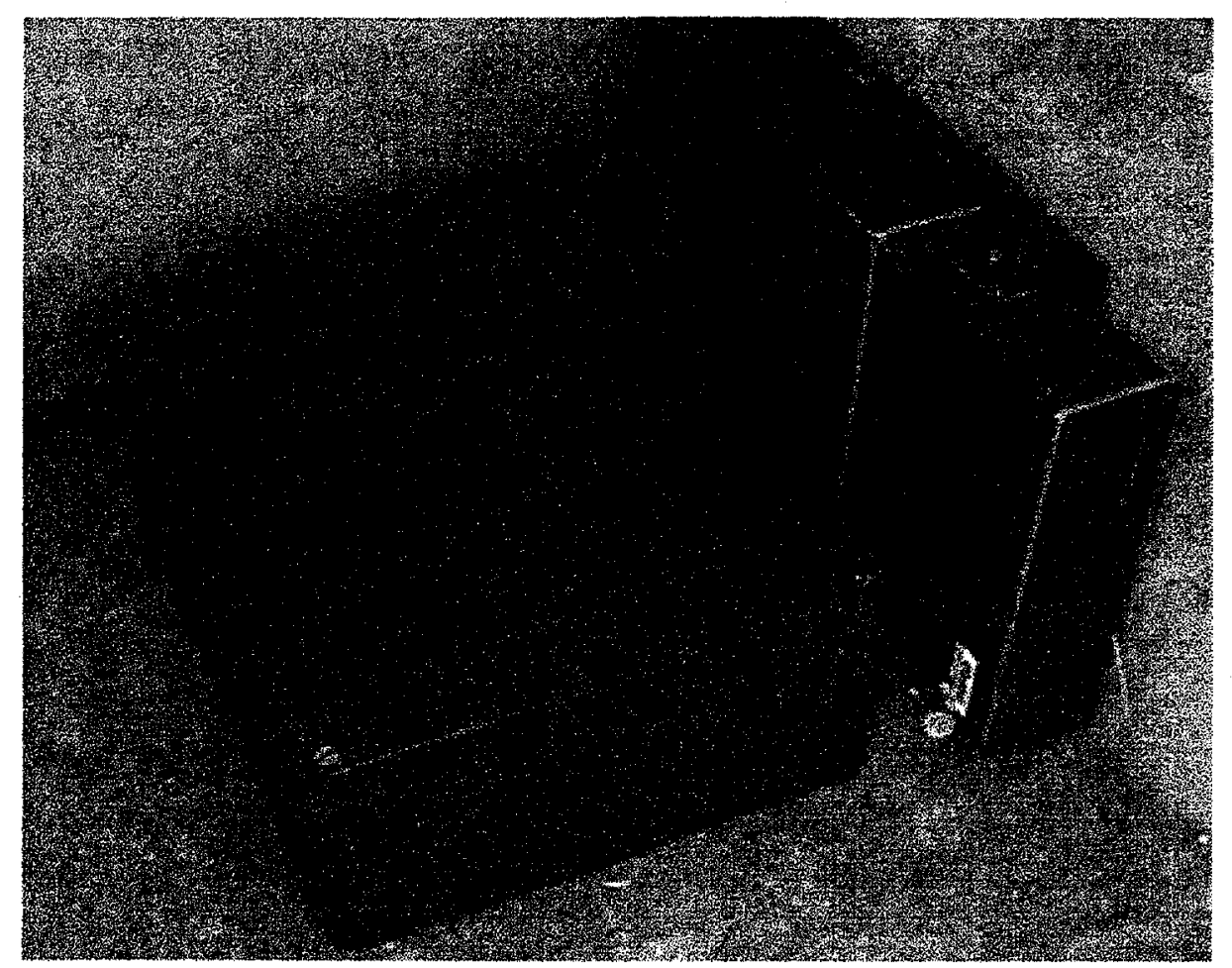

Figure 6 
The other problem that we encountered was where to mount the GPS antennas on the vehicles. The antennas that came with the GPS units were intended to be temporarily mounted using a built in magnetic base. The units also came with a mast mount which is great for mounting the antenna on a boat mast. The mast mount adapter mounted to the bottom of the antenna with four screws. The four screw mount was used to mount the antennas on top of the emergency light bar on each vehicle. Figure 7 is a photo of the antenna mounted on one of the light bars.

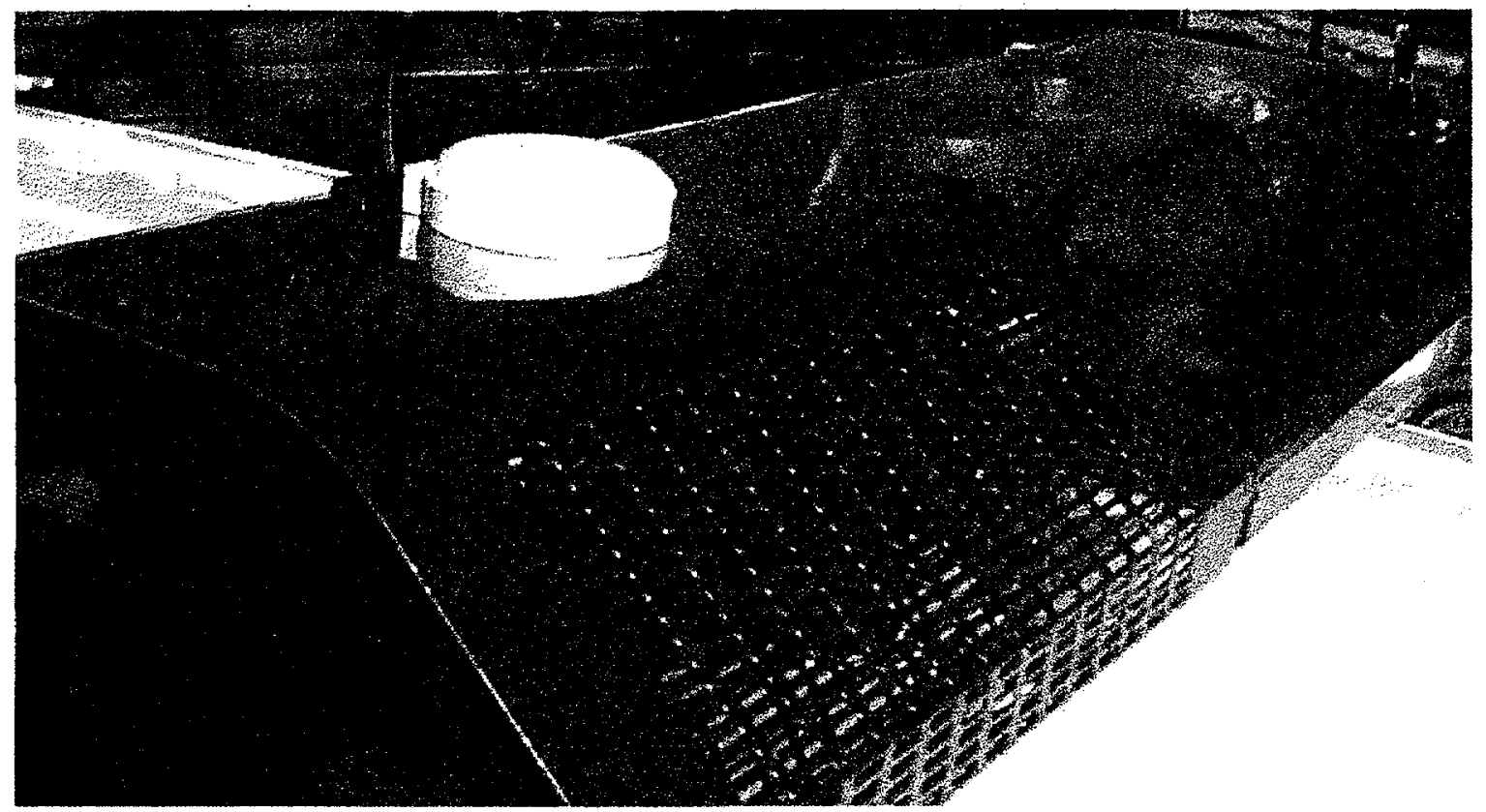

Figure 7

Standard VHF vehicle radio antennas were mounted to each vehicle for the VHF radio. The power for the package was hooked up so that the ignition switch controlled the power. The AVL system operates whenever the vehicle's ignition is activated.

\section{Base Station Configuration}

The Base station at LANL is set up at the EOC in the radio dispatch center, where it is in full view of the dispatcher at all times (see Figure 8). The monitor provides real time tracking of the two EM\&R vehicles. Figure 9 is an example of the computer monitor output. The two vehicles are labeled EMRI and EMR2 which can be seen in Figure 9. The box on the upper right indicates the location of EMR1 on its last transmission. 


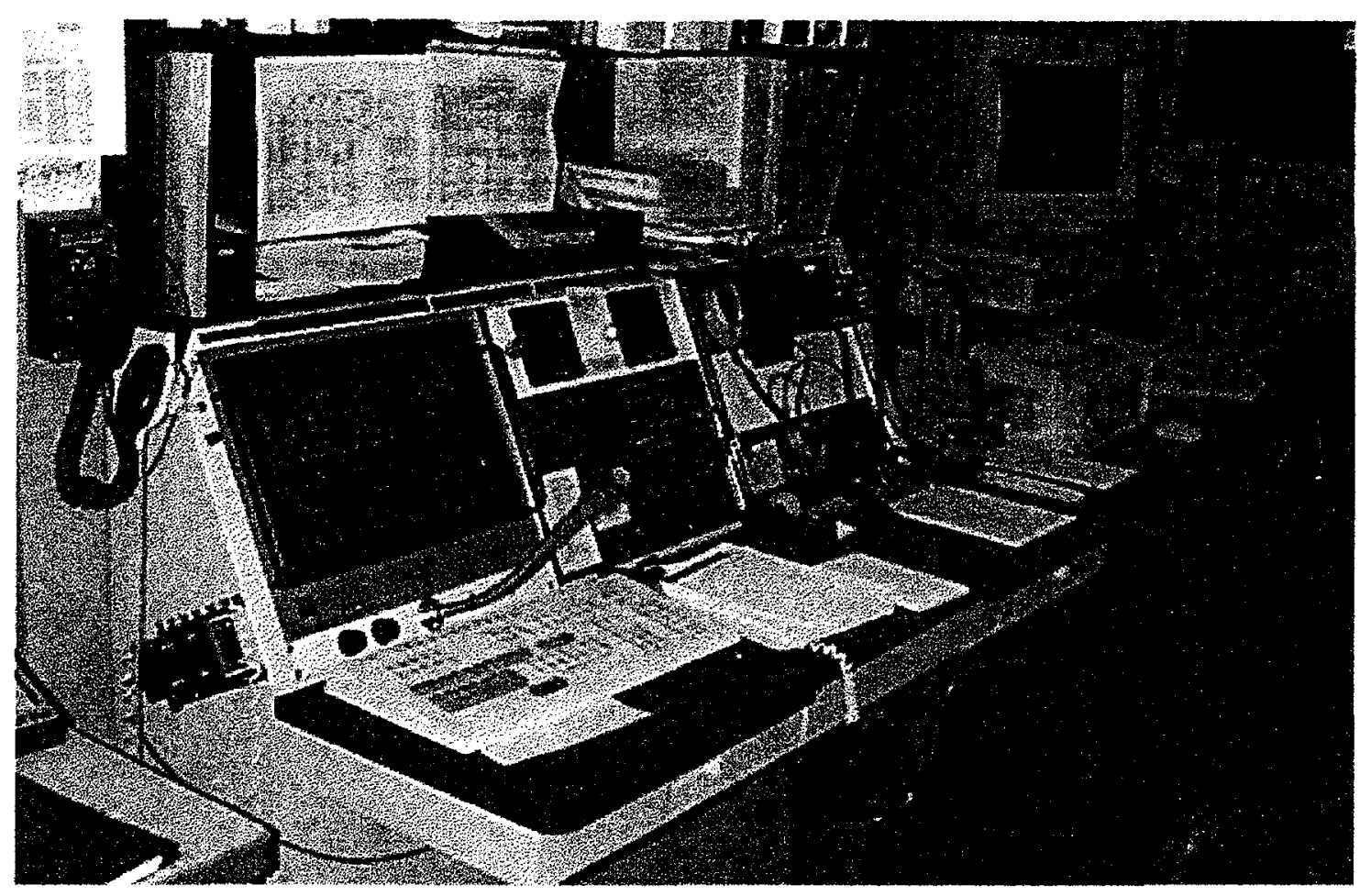

Figure 8

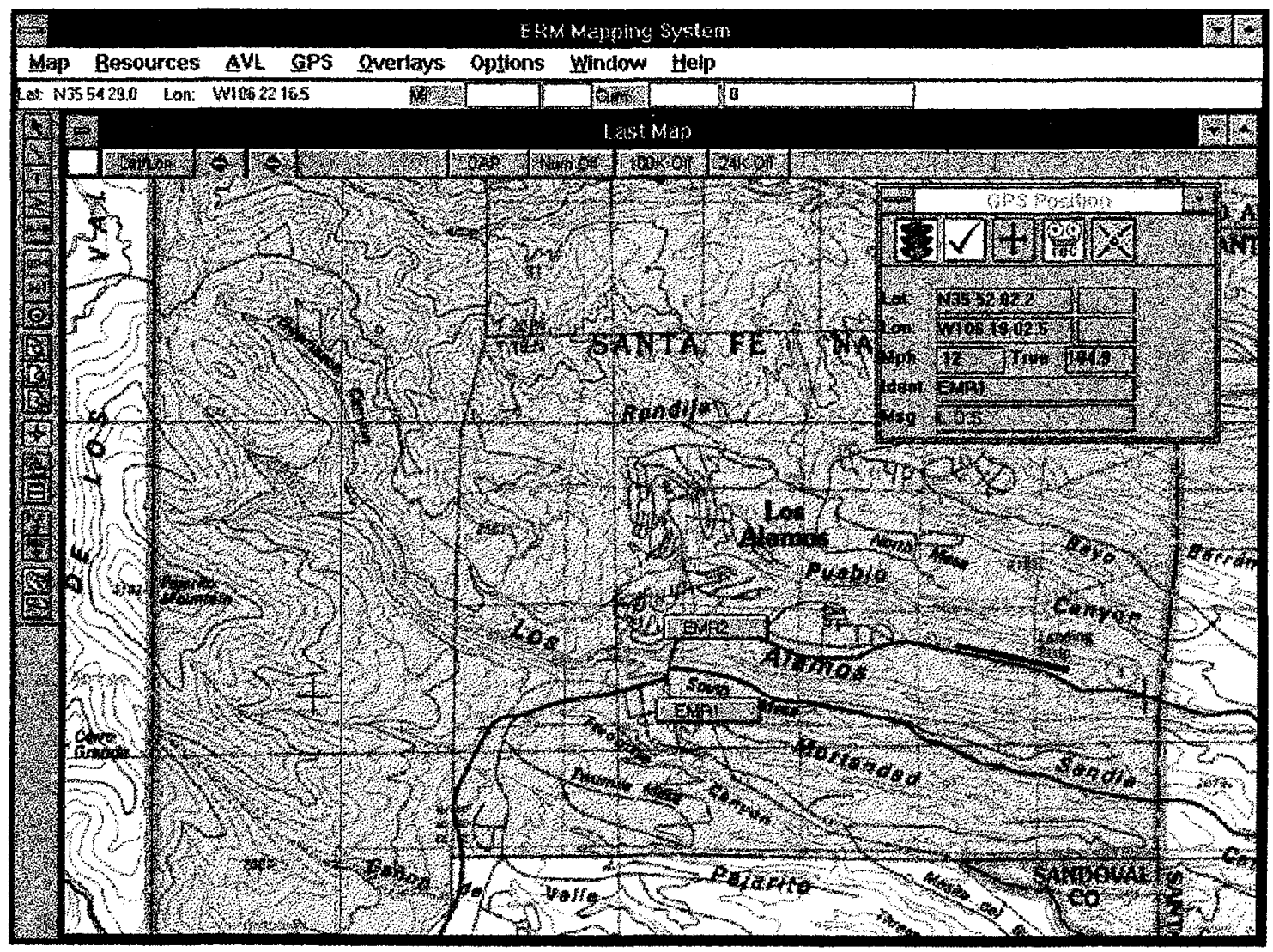

Figure 9 


\section{Conclusion}

The system described in this paper is in operation today and is providing location information to LANL's EM\&R EOC. The two emergency vehicles have been in operation for two months and continue to operate without any problems. This project met all of the requirements for a low cost reliable AVL system.

\section{Acknowledgments}

Special Thanks to the following people who made this project possible. Doug Tuggle, FSS-20, for providing coordination in vehicle availability. Dave Howard, FSS-20, for computer support and photographs. Abedon Ortiz Jr. and Pedro W. Romero, CIC-4, for installing the boxes and communications equipment on both of the emergency vehicles. Midshipman Matthew W. Farr, USN, for assisting in drawings and assembly. 
Appendix 1 Equipment Suppliers

A. GPS Unit

Model : Trimble Scout Master

Trimble Navigation LTD.

645 N. Mary Ave.

Sunnyvale, California 94086

(408)481-8000

B. Modem

Model : Kantronic WM1200

Kantronic RF Data Communications Specialists

1202 E. 23rd Street

Lawrence, Kansas 66046

(913) $842-7745$

C. Radio

Model : Motorola Maxtrac

Motorola, Inc.

1301 E. Algonquin Road

Schaumburg, Illinois 60196

(800) 247-2346

D. Software

Model: E.R.M. GPS Software

E.R.M., Inc.

P.O. Box 770039

Steamboat Springs, Colorado 80477

(800) 878-6277 